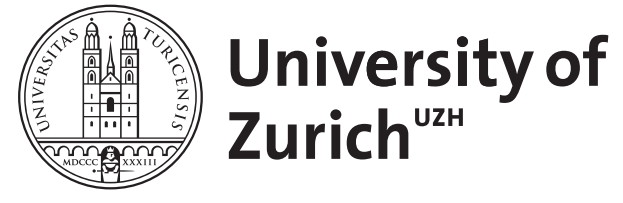

\title{
Qualitätszirkel Oberthurgau
}

Gnädiger, M ; Nadig, M ; Buff, R ; Haug, B ; Rohrbasser, A

\begin{abstract}
Seit mehr als 10 Jahren bietet unser hausärztliches Netzwerk im Oberthurgau regelmässige offene Qualitätszirkel-Sitzungen an. In diesem Beitrag stellen wir unseren QZ vor. Die Daten stützen sich auf die Analyse aller QZ-Protokolle der Jahre 2008 bis 2012 sowie auf eine strukturierte Umfrage (94\% Rücklauf), die unter den 18 Teilnehmern und dem Fachreferenten des QZOst vom 2.10.2012 zum Thema «Nähen, kleben, leimen oder klammern? / Wundversorgung in der hausärztlichen Praxis» durchgeführt wurde.
\end{abstract}

Posted at the Zurich Open Repository and Archive, University of Zurich ZORA URL: https://doi.org/10.5167/uzh-80846

Journal Article

Published Version

Originally published at:

Gnädiger, M; Nadig, M; Buff, R; Haug, B; Rohrbasser, A (2013). Qualitätszirkel Oberthurgau. PrimaryCare, 13(8):142-143. 


\section{Qualitätszirkel Oberthurgau}

\begin{abstract}
Seit mehr als 10 Jahren bietet unser hausärztliches Netzwerk im Oberthurgau regelmässige offene Qualitätszirkel-Sitzungen an. In diesem Beitrag stellen wir unseren $\mathrm{QZ}$ vor. Die Daten stützen sich auf die Analyse aller QZ-Protokolle der Jahre 2008 bis 2012 sowie auf eine strukturierte Umfrage (94\% Rücklauf), die unter den 18 Teilnehmern und dem Fachreferenten des QZOst vom 2.10.2012 zum Thema «Nähen, kleben, leimen oder klammern? / Wundversorgung in der hausärztlichen Praxis» durchgeführt wurde. ${ }^{1}$
\end{abstract}

Seit bald 20 Jahren werden in der Schweiz Kurse für Qualitätszirkel (QZ)-Moderatoren angeboten. Die Kriterien, die einen QZ von einem Treffen der «Vier beim Bier» unterscheiden, wurden von der Delegiertenversammlung von «Hausärzte Schweiz» am 20. Mai 2011 [1] festgelegt:

- 8 QZ pro Jahr, der Besuch von mindestens 6 wird erwartet;

- Dauer $1 \frac{1}{2}$ bis 2 Stunden;

- Leitung durch ausgebildete Moderatoren, Assistenz durch CoModeratoren;

- definierte Sitzungsziele;

- Protokolle;

- regelmässige Prozess- und Ergebnis-Evaluation;

- hierarchische Gleichstellung aller Mitglieder.

Die im QZ verwendeten Methoden umfassen anerkannte Schritte wie Audit (kompletter Qualitätszyklus) [2] und Feedback mit und ohne Besuche der Praxis, den Einbezug lokaler Wissensexperten und die Arbeit in einer Workshop-ähnlichen Atmosphäre - alles Schritte, die erwiesenermassen Veränderungen bewirken können [3-7]. Der Moderator hält die Gruppe auf Kurs und bezieht alle Mitglieder mit ein. Moderatoren haben mit ihrer Kompetenz grossen Einfluss auf das Resultat [8]. OZ sind also komplexe, facettenreiche Interventionen, die neben der Wissensvermittlung eine Interaktion innerhalb der Gruppe beinhalten. Zudem beziehen sie eine Kontrolle der Ergebnisse mit ein und wirken damit auch nachhaltig [9].

\section{Einige Daten zu unserem Qualitätszirkel}

Der OZ Oberthurgau besteht aus zwei separaten, sich ergänzenden Einheiten, dem QZ «Oberthurgau-Ost» und «Oberthurgau-West». Die Teilnahme ist freiwillig, eine Entschuldigung ist erwünscht. Von unserem $\mathrm{QZ}$ nicht übernommen wurden frühere Kriterien [10] wie eine feste Gruppenzusammensetzung oder eine verbindliche Teilnahme. Die QZ sind offen für alle Eingeladenen. Auch die interessierten Mitglieder des Netzwerks Rorschach, die in nicht dessen geschlossenen QZ eingebunden sind, werden eingeladen.

Qualitätszirkel Oberthurgau;

Institut für Hausarztmedizin und Versorgungsforschung der Universität Zürich

Die detaillierten Resultate dieser Umfrage sind erhältlich auf www.forum-hausarztmedizin.ch («PrimaryCare-Diskussionen»).
Durchschnittlich fanden 2008 bis 2012 in beiden QZ jährlich 7 bis 9 Sitzungen mit je 12 bis 14 Teilnehmenden statt, bei einer Spannweite von 3 bis 22. Bei kleineren Treffen melden sich auch die zurückhaltenden Teilnehmenden vermehrt zu Wort. Neben Hausärzten nahmen gelegentlich auch Dermatologen, Psychiater, orthopädische Chirurgen und Gynäkologen teil, auch wenn sie nicht als Referenten eingeladen waren.

Rund die Hälfte der Teilnehmer des 2.10.12 deklariert, fast immer teilzunehmen und aktiv mitzudiskutieren. Die übrigen kommen, wenn Thema und Zeitpunkt passen und sie den Termin nicht vergessen - und mehr zum Zuhören.

Unser Themenspektrum deckt die ganze Allgemeinmedizin ab. Das Format unserer Qualitätszirkel ähnelt jenem der Seminare an unseren grossen Kongressen mit einem Fachreferenten und einem hausärztlichen Moderator. Im QZ jedoch kennen sich die Teilnehmer persönlich.

Meist laden wir einen externen Fachexperten zum jeweiligen QZThema ein. Nach einer kurzen Vorstellungsrunde findet eine Praktiker-Diskussion statt. Der Referent hört bei diesem Diskurs zu, ohne sich selber zu äussern. Wenn sich das Gespräch unter «Peers» erschöpft hat, übernimmt er es, offene Fragen zum Thema zu beantworten und gegebenenfalls auch einige Folien zu präsentieren. Eine Co-Moderation zu zweit pflegen wir nur beim Thema «critical incident reporting» (CIRS) zu machen.

Wir führen keine Audits mit einem vollständigen Qualitäts-Zyklus und auch keinen Feedback durch. Diese können in bestimmten Bereichen (z.B. Diabetes-Behandlung) sehr eindrückliche Verhaltensänderungen bei Ärzten bewirken [3], wie sich z.B. in Ländern wie Dänemark oder Schweden zeigt. Wahrscheinlich wäre die Motivation zu Audit und Feedback für verschiedene chronische Erkrankungen in der Schweiz grösser, wenn den Hausärzten auf Anfrage ohne Mühe valide Daten zur Verfügung gestellt würden.

Gemäss zwei Managed-Care-Verträgen müssen wir 10 QZ-Sitzungen pro Jahr anbieten, davon mindestens je einen zum Thema «Wirtschaftlichkeit» und CIRS (critical incident reporting). Da wir zwei parallele offene $\mathrm{QZ}$ betreiben, erfüllen wir diese Vertragsbedingung.

Die Einladung erfolgt per E-Mail-Versand über unser Dienstleistungs- und Kompetenzzentrum «eastcare». Es wird nach jeder Veranstaltung ein Protokoll verfasst, welches auf demselben Weg den Mitgliedern zugestellt wird; diese Protokolle bleiben anschliessend im internen Mitgliederbereich von «eastcare» aufgeschaltet. QZ-Teilnehmer, die unserem Ärztenetzwerk angehören, werden aus der Netzwerkkasse mit 150.- Fr. pro Teilnahme entschädigt. Nicht-Netzwerkärzte sowie die Ärzte aus dem Netzwerk Rorschach erhalten keine Entschädigung. Der verantwortliche Moderator erhält zusätzlich Fr. 150.- für die Organisation, die Leitung und die Verfassung des Protokolls. Die Terminplanung der ganzen OZSerie sowie die Suche nach geeigneten Themen und allfälligen Referenten werden von den Leitern ehrenamtlich geleistet.

Für die Aufwendungen des QZ haben wir ein Pharma-Dual-Sponsoring für jeweils eine ganze Jahresserie. Davon werden die Saalmiete, die Konsumation und ein kleiner Steh-Apéro nach dem QZ beglichen. Auch das Referenten-Honorar, sofern benötigt, wird so entschädigt. 


\section{Grosse Zufriedenheit der Teilnehmenden und Referenten}

Die Umfrage unter den Teilnehmenden und beim Fachexperten des Qualitätszirkels vom 2.10.2012 zum Thema der Wundversorgung in der Praxis ergab eine grosse Zufriedenheit mit diesem spezifischen QZ und dem QZ im Allgemeinen. Eine Nachbefragung nach 3 Monaten bestätigte diesen Eindruck. Die Fragen betrafen: Themenwahl und Inhalte der QZ-Sitzungen, die Exaktheit und Nützlichkeit der Protokolle sowie die durch die QZ-Teilnahme ausgelösten nachhaltigen Verhaltensänderungen. Nach dem QZ Wundversorgung hat beispielsweise ca. die Hälfte der Hausärzte Veränderungen eingeführt. Auch der Referent war sehr zufrieden. Ein positiver Nebeneffekt war, dass sich die Hausärzte und der chirurgische Fachexperte einmal persönlich kennen lernen konnten. Unser offener Qualitätszirkel hält sich am Fortbildungs-«Markt». Seit Jahren ist er eine erfolgreiche Veranstaltung. Dies zeigt, dass er einem Bedürfnis entspricht und den Hausärzten in der angebotenen Form gefällt. Er besteht aus einem inneren Kreis von «Dauerteilnehmern» und einer grösseren Gruppe von gelegentlichen Besuchern («à la carte»-Teilnehmer). Aufgrund der positiven Rückmeldungen werden wir so weitermachen, auch wenn unser QZ nicht «der reinen Lehre» entspricht.

Die Autoren deklarieren, mit diesem Artikel keine kommerziellen Interessen zu verfolgen.

\section{Literatur}

1 Brühwiler J. Qualitätszirkel als Grundelement der Qualitätsentwicklung in der Hausarztpraxis. PrimaryCare. 2011;11 (21):365.

2 Rohrbasser A. In den QZ - aus dem QZ. PrimaryCare. 2012;12(19):375-6.

3 Ivers N, Jamtmedt G, Flottorp S, et al. Audit and feedback: effects on professional practice and healthcare outcomes. Cochrane Database. 2012.

4 Forsetlund L, Bjorndal A, Rashidian A, et al. Continuing educations meetings and workshops: effects on professional practice and health outcomes. Cochrane Database. 2009.

5 Flodgren G, Parmelli E, Boumit G, et al. Local opinion leaders: effects on professional practice and healthcare outcomes. Cochrane Database. 2011.

6 O'Brian MA, Rogers S, Jamtvedt G, et al. Educational outreach visits: effects on professional practice and healthcare coutcomes. Cochrane Database. 2007.

7 Elewyn GGT, MacFarlane F. Groups. A guide to small group work in healthcare, management, education and research. Radcliff Medical Press: 2004.

8 Baskerville NB, Liddy C, Hogg W. Systematic review and meta-analysis of practice facilitation within primary care settings. Ann Fam Med. 2012;10: 63-74.

9 Davis DA, Thomson MA, Oxman AD, et al. Changing physician performance. A systematic review of the effect of continuing medical education strategies. JAMA. 1995;274(9):700-5.

10 Dahinden A. Qualitätszirkel (QZ) in Deutschland, Österreich und der Schweiz. PrimaryCare. 2003;3:1020-2.

Korrespondenz:

Dr. med. Markus Gnädinger

Facharzt für Innere Medizin

Birkenweg 8

9323 Steinach

markus.gnaedinger[at]hin.ch 